\title{
Metal-rich infall onto the inner disk through the interaction between bulge winds and gaseous halos
}

\author{
Takuji Tsujimoto ${ }^{1}$ and Kenji Bekki ${ }^{2}$ \\ ${ }^{1}$ National Astronomical Observatory, Mitaka-shi, Tokyo 181-8588, Japan \\ email: taku.tsujimoto@nao.ac.jp \\ ${ }^{2}$ School of Physics, University of New South Wales, Sydney 2052, NSW, Australia \\ email: bekki@phys.unsw.edu .au
}

\begin{abstract}
We demonstrate for the first time that gaseous halos of disk galaxies can play a vital role in recycling metal-rich gas ejected from the bulges and thus in promoting the chemical evolution of the disks. Our numerical simulations show that metal-rich bulge winds can be accreted onto the thin disks owing to hydrodynamical interaction between the gaseous ejecta and the gaseous halos. Accordingly, we anticipate that chemical abundances of the inner disk stars are significantly influenced by the enriched winds. About $\sim 1 \%$ of gaseous ejecta from the bulges can be accreted onto the middle disk corresponding to the sun's position. We discuss these results in the context of the origin of super metal-rich stars in the solar neighborhood as well as an observed flattening of the abundance gradient in the Galactic disk.
\end{abstract}

Keywords. Galaxy: bulge, Galaxy: disk, Galaxy: evolution, stars: abundances

\section{Evidence for metal-rich infall}

\section{1. solar neighborhood}

The metal-rich end of the abundance distribution function (ADF) of solar neighborhood stars extends at least to $[\mathrm{Fe} / \mathrm{H}] \sim+0.2$, and the fraction of stars with $[\mathrm{Fe} / \mathrm{H}]>0$ is roughly $20 \%$. Spectroscopic observations of elemental abundances for metal-rich disk stars (Feltzing \& Gustafsson 1998; Bensby et al. 2005) have confirmed that chemical enrichment in the solar neighborhood has continuously proceeded until $[\mathrm{Fe} / \mathrm{H}] \sim+0.4$. Tsujimoto (2007) has claimed that the simultaneous reproduction of both the presence of stars with $[\mathrm{Fe} / \mathrm{H}]>+0.2$ and $[\mathrm{Fe} / \mathrm{H}]_{\text {peak }}<0$ of the ADF is hard to realize through the conventional scheme of local enrichment via low-metallicity gaseous infall from the halo, and that the presence of supersolar stars is crucial evidence for enrichment by winds from the bulge. It should be stressed that the upturning feature of $[\mathrm{Mg} / \mathrm{Fe}]$ for $[\mathrm{Fe} / \mathrm{H}]>$ 0 strongly implies the enrichment by winds having an enhanced $\mathrm{Mg} / \mathrm{Fe}$ ratio, since this feature can not be explained by radial mixing (e.g., Roškar et al. 2008).

2. time evolution of the Galactic metallicity gradient

The radial metallicity gradient roughly from $R_{\mathrm{GC}}=4$ to $R_{\mathrm{GC}}=14 \mathrm{kpc}$ has flattened out in the last several Gyr with a change in a slope of $\sim-0.1 \mathrm{dex} \mathrm{kpc}^{-1}$ to $\sim-0.05$ dex $\mathrm{kpc}^{-1}$ (Chen et al. 2003; Maciel et al. 2006). Galactic Chemical evolution (GCE) models predict contradictory time evolution of the metallicity gradient, i.e., a steepening (Chiappini et al. 2001) or a flattening (Hou et al. 2000). Putting aside these contradictions, existing GCE models predict a monotone increase in abundances for each region, and the predicted gradient change is small compared with the observations. We found the steep relic gradient observed in old open clusters while a shallower gradient for Cepheids. 
We note that the gradient became shallower owing to a decrease in metallicity in the inner regions, with a corresponding increase in the outer region. These features must come from the phenomenon that early wind enrichment sets up the initially steep gradient.

\section{Hydrodynamical simulations}
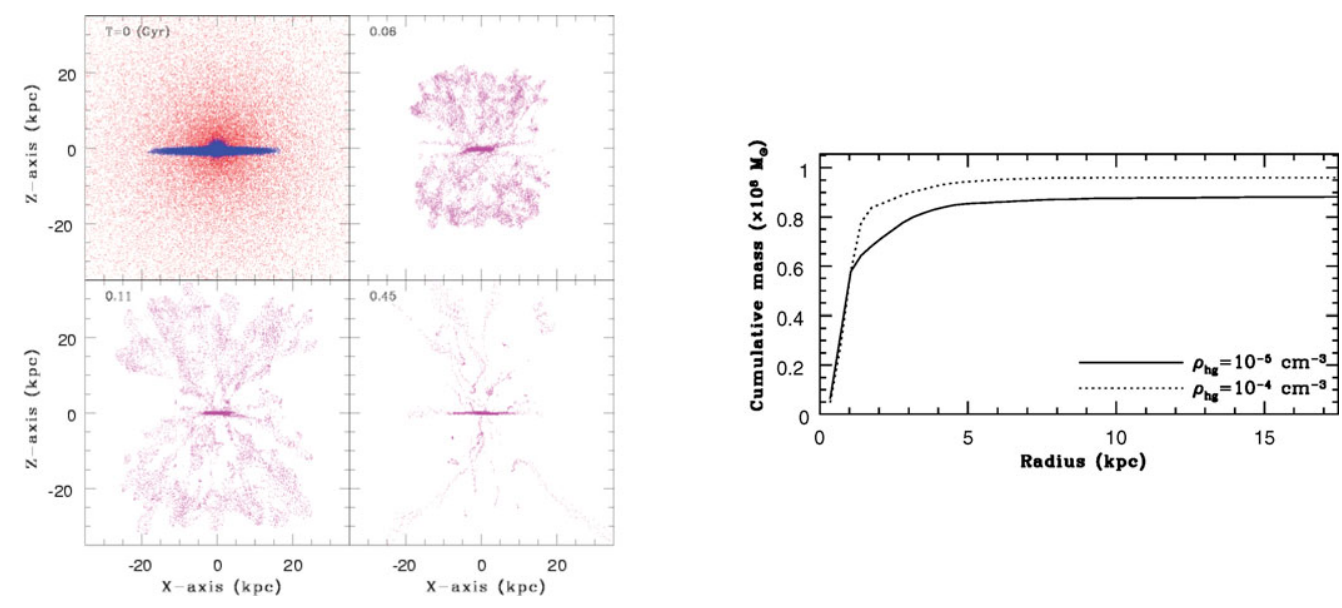

We numerically investigate the dynamical evolution of gas ejected from bulges in disk galaxies using our own GRAPE-SPH codes. The left panel of figure shows how gas ejected from a bulge evolves with time during hydrodynamical interaction between the gas and the halo gas in a disk galaxy. Although a significant fraction of the gas once escapes from the bulge $(T=0.11 \mathrm{Gyr})$, most of the gas can be finally returned to the disk plane owing to gaseous pressure from the halo and the disk $(T=0.45 \mathrm{Gyr})$. The bulge gas particles initially in the central region of the bulge can interact so strongly with the disk gas particles immediately after the ejection that a thin disk can be formed from the bulge gas at $R<3 \mathrm{kpc}$ for a short time scale $(T=0.06 \mathrm{Gyr})$. The bulge gas particles ejected from the bulge regions with larger $|z|$ can be later accreted onto the outer part of the disk and consequently can rotate around the center of the galaxy. The mean orbital eccentricity of bulge gas particles in the solar neighborhood is 0.08 in this model, which means that the orbits become almost circular, because the particles have acquired orbital angular momentum during hydrodynamical interaction with the disk gas.

The right panel shows that almost $80 \%$ of the bulge gas can be accreted onto the inner disk region with $R \leqslant 3 \mathrm{kpc}$ in the model with $\rho_{\mathrm{hg}}=10^{-5} \mathrm{~cm}^{-3}$ where $\rho_{\mathrm{hg}}$ denotes the gas density of halo. This preferential accretion can be clearly seen also in the model with $\rho_{\mathrm{hg}}=10^{-4} \mathrm{~cm}^{-3}$.

\section{References}

Bensby, T., Feltzing, S., Lundström, I., \& Ilyiin, I. 2005, $A \& A$, 433, 185

Chen, L., Hou, J. L., \& Wang, J. J. 2003, AJ, 125, 1397

Chiappini, C., Matteucci, F., \& Romano, D. 2001, ApJ, 554, 1044

Feltzing, S. \& Gustafsson, B. 1998, A\&AS, 129, 237

Hou, J. L., Prantzos, N., \& Boissier, S. 2000, A\&A, 362, 921

Maciel, W. J., Lago, L. G., \& Costa, R. D. D. 2006, A\&A, 453, 587

Roškar, R., Debattista, V. P., Quinn, T. R., Stinson, G. S., \& Wadsley, J. 2008, ApJ, 684, L79

Tsujimoto, T. 2007, ApJ, 665, L115 